\title{
Identification of genomic regions affecting grain peroxidase activity in bread wheat using genome-wide association study
}

Zhengfu Zhou ${ }^{1,2 \dagger}$, Huiyue Guan ${ }^{1,2 \dagger}$, Congcong Liu ${ }^{1,3}$, Ziwei Zhang ${ }^{3}$, Shenghui Geng ${ }^{1,2}$, Maomao Qin ${ }^{1}$, Wenxu Li ${ }^{1}$, Xia Shi ${ }^{1}$, Ziju Dai ${ }^{1}$, Zhensheng Lei ${ }^{1,2,3}$, Zhengqing Wu ${ }^{1,2^{*}}$, Baoming Tian ${ }^{1,2^{*}}$ and Jinna Hou ${ }^{1^{*}}$

\begin{abstract}
Background: Peroxidase (POD) activity plays an important role in flour-based product quality, which is mainly associated with browning and bleaching effects of flour. Here, we performed a genome-wide association study (GWAS) on POD activity using an association population consisted with 207 wheat world-wide collected varieties. Our study also provide basis for the genetic improvement of flour color-based quality in wheat.
\end{abstract}

Results: Twenty quantitative trait loci (QTLs) were detected associated with POD activity, explaining 5.59-12.67\% of phenotypic variation. Superior alleles were positively correlated with POD activity. In addition, two SNPs were successfully developed to KASP (Kompetitive Allele-Specific PCR) markers. Two POD genes, TraesCS2B02G615700 and TraesCS2D02G583000, were aligned near the QTLs flanking genomic regions, but only TraesCS2D02G583000 displayed significant divergent expression levels $(P<0.001)$ between high and low POD activity varieties in the investigated association population. Therefore, it was deduced to be a candidate gene. The expression level of Traes $C$ S2D02G583000 was assigned as a phenotype for expression GWAS (eGWAS) to screen regulatory elements. In total, 505 significant SNPs on 20 chromosomes (excluding 4D) were detected, and 9 of them located within $1 \mathrm{Mb}$ interval of TraesCS2D02G583000.

Conclusions: To identify genetic loci affecting POD activity in wheat grain, we conducted GWAS on POD activity and the candidate gene TraesCS2D02G583000 expression. Finally, 20 QTLs were detected for POD activity, whereas two QTLs associated SNPs were converted to KASP markers that could be used for marker-assisted breeding. Both cisand trans-acting elements were revealed by eGWAS of TraesCS2D02G583000 expression. The present study provides genetic loci for improving POD activity across wide genetic backgrounds and largely improved the selection efficiency for breeding in wheat.

Keywords: Wheat, POD activity, GWAS, Flour color, Wheat quality

*Correspondence: wzhqfy@163.com; tianbm@zzu.edu.cn; houjinna@hnagri. org.cn

†Zhengfu Zhou and Huiyue Guan contributed equally to this work. ${ }^{1}$ Henan Institute of Crop Molecular Breeding, Postgraduate T \& R Base of Zhengzhou University, Henan Academy of Agricultural Sciences, Zhengzhou 450002, China

${ }^{2}$ Agronomy college, Zhengzhou University, Zhengzhou 450001, China

Full list of author information is available at the end of the article

\section{Background}

Flour color is an important parameter for evaluating the end-use quality of flour products, such as noodles and bread [1]. Multiple factors affect flour color, including yellow pigment content, oxidase activity, wheatgrain color, wet gluten content, water absorption, wheat bran content, and protein content. Yellow pigment content, which completely associated with Phytoene Synthase 1 (Psy1) gene, is one of the important 
factors influencing flour yellowness in wheat. Selection of Psy1 lose function allele would improve flour color through altering carotenoid content [2-4]. Higher protein content can also affect the color of flour products but mainly through darkening dough color via effects on grain hardness, flour texture, and water absorption during the milling process [5].

In addition, colorless precursor substances in flour could produce colored substances through the enzymatic reaction of oxidases, thus affecting flour color. Typically, wheat grains contain three oxidase types: peroxidase (POD), polyphenol oxidase (PPO), and lipoxygenase (LOX). Of these, heme-containing PODs have a wide range of distribution in higher plant cells [6]. The first protein sequence of POD was determined in 1979 [7], and differences in primary structure have resulted in the plant POD super-family being categorized into class I, II, and III [8]. Class I is a group of intracellular enzymes that are extensively present in plants, bacteria, and yeast. This class includes cytochrome $\mathrm{C}$ peroxidase $(\mathrm{CCP})$, a soluble protein in the electron transport chain of mitochondria that prevents damage from toxic peroxides; ascorbate peroxidase (AP), which mainly functions to expend hydrogen peroxide in the chloroplast and cytosol of higher plants [9]; and bacterial catalaseperoxidase, which protects cells under oxidative stress [10]. Class II contains four conserved disulfide bonds and two conserved calcium binding sites. They are usually extracellular fungal peroxidases and are assigned as lignin peroxidase or ligninase because they catalyze lignin depolymerization [11]. Class III includes classic and plant-specific peroxidases secreted into the vacuole and cell wall [12], with tissue-specific functions. Previous studies have shown that PODs are involved in various aspects of plant physiology, such as oxidizing compounds with hydrogen peroxide as an electron acceptor, defending against insect attack, generation and detoxification of active oxygen forms, lignin formation, and cell wall biosynthesis [13-15].

Many wheat-grain tissues contain POD, including the epidermis, seed coat, embryo, and endosperm. As a redux enzyme, POD can oxidize ferulic acid and other major phenolic acids, producing chromophoric groups and brown substances [15-17]. High POD activity may darken flour and subsequently result in undesirable pasta color $[17,18]$. Indeed, the brown index of pasta products and POD activity are significantly correlated $(r=0.84-$ 0.97) $[19,20]$. However, when $3000 \mathrm{U}$ POD and 815$1630 \mathrm{U}$ LOX were added to $100 \mathrm{~g}$ flour, the dough was completely bleached, indicating that POD could replace benzoyl peroxide as a bleaching agent. Furthermore, POD causes less damage to the flavor of pasta products than LOX when used as the main food additive $[21,22]$.
Genotype is the primary influencer of POD activity [23-25], although environmental factors also play a role [26] . Previous studies have revealed that genetic variance in POD activity accounts for $90 \%$ of total variance [27]. Common wheat has significantly higher POD activity than durum wheat $(P<0.05)$ [28]. Among common wheat varieties, POD activity is fairly variable, differing by $3-10$ times [29]. These results suggest that POD activity could be altered through manipulating desired alleles by gene pyramiding. Common wheat contains three subgenomes $(\mathrm{A}, \mathrm{B}$, and $\mathrm{D})$, and genes are usually triplicated. Therefore, many copies of POD genes are located on the corresponding homologous regions, distributed throughout wheat A, B, and D genomes [30]. Using CS-nullitetrasomic lines, researchers reported POD genes on chromosomes 2A, 2B, 2D, 4B, 7A, and 7D [31]. Quantitative trait loci (QTL) analysis on POD activity using a RIL population (204 lines derived from crossing Doumai and Shi4185) revealed three significant QTLs, QPod.caas$3 A L$, QPod.caas-4BS, and QPod.caas-5AS, explaining $5.3 \sim 21.2 \%$ of phenotypic variation. Functional markers have been developed from the genomic DNA sequence of wheat POD genes [32]. In addition, research that used Gramineae collinearity identified QTL for POD activity through POD genes in rice-specifically, relevant wheat QTL were located on the homologous region of chromosomes 3 and 7 , with the former being particularly important $[33,34]$.

Numerous studies have investigated how LOX and PPO activity are linked to flour color [22], but few have examined the genetic basis of POD activity in wheat association population. To better understand POD genetic diversity in wheat varieties, we conducted a genome-wide association study (GWAS) to detect QTLs associated to grain POD activity in a panel of 207 wheat varieties, and predicted candidate genes. In addition, we developed KASP molecular markers from SNPs located in major loci and evaluated their correlation with POD activity. Our results will greatly contribute to the improvement of flour color and provide theoretical support for advancing wheat molecular breeding.

\section{Results}

\section{Phenotypic variation in the association population}

Peroxidase activity was investigated in an association population of 207 varieties planted in three locations. Generally, mean POD activity in the three environments was $753.75 \mathrm{U} \cdot \mathrm{min}^{-1} \cdot \mathrm{g}^{-1}$. The highest average POD activity was $889.99 \mathrm{U} \cdot \mathrm{min}^{-1} \cdot \mathrm{g}^{-1}$ in Kaifeng (KF), and the lowest average was $575.94 \mathrm{U} \cdot \mathrm{min}^{-1} \cdot \mathrm{g}^{-1}$ in Shangqiu (SQ) (Fig. 1a, Table 1). Maximum POD activity was 1718.42 $\mathrm{U} \cdot \mathrm{min}^{-1} \cdot \mathrm{g}^{-1}$ in Yuanyang (YY), where the range was $186.00 \sim 1718.42 \mathrm{U} \cdot \mathrm{min}^{-1} \cdot \mathrm{g}^{-1}$ (Table 1). Minimum 

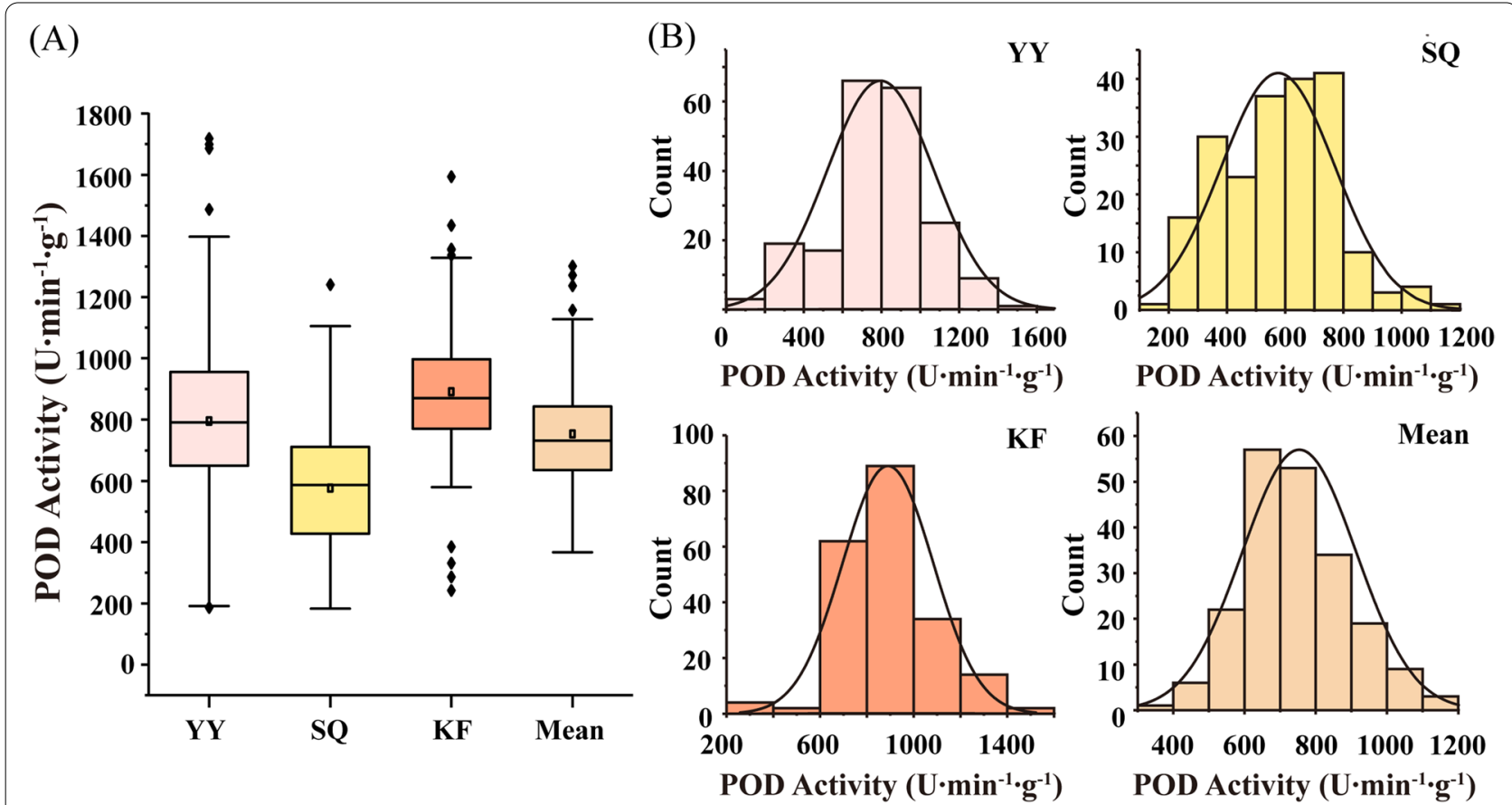

Fig. 1 Distribution of POD activity in the association population under different environments. a Boxplot of POD activity in Yuanyang (YY), Shangqiu (SQ), and Kaifeng (KF), plus the mean of all three environments. $\mathbf{b}$ Distribution plots of POD activity in the three environments and their mean

Table 1 Statistical analyses of POD activity in the association population

\begin{tabular}{|c|c|c|c|c|c|}
\hline Environment $^{a}$ & $\operatorname{Mean} \pm S D^{b}\left(U \cdot \min ^{-1} \cdot g^{-1}\right)$ & Range & Kurtosis & Skewness & $\mathrm{CV}^{\mathrm{C}}(\%)$ \\
\hline YY & $795.34 \pm 275.73$ & $186.00 \sim 1718.42$ & 1.04 & 0.24 & 34.67 \\
\hline SQ & $575.94 \pm 194.84$ & $182.75 \sim 1240.97$ & 0.01 & 0.25 & 33.83 \\
\hline KF & $889.99 \pm 194.35$ & $242.72 \sim 1593.75$ & 1.53 & 0.30 & 21.84 \\
\hline Mean & $753.75 \pm 160.00$ & $366.93 \sim 1301.30$ & 0.75 & 0.70 & 21.23 \\
\hline
\end{tabular}

${ }^{a}$ Environment: YY (Yuanyang), SQ (Shangqiu), KF (Kaifeng);

b SD Standard deviation

c CV Coefficient of variation

POD activity was $182.75 \mathrm{U} \cdot \mathrm{min}^{-1} \cdot \mathrm{g}^{-1}$ in Shangqiu (SQ), where the range was $182.75 \sim 1240.97 \mathrm{U} \cdot \mathrm{min}^{-1} \cdot \mathrm{g}^{-1}$. In all three environments, POD activity exhibited continuous and wide variation that was close to a normal distribution (Fig. 1b). The coefficient of phenotypic variation ranged from 21.84 to $34.67 \%$ (Table 1). Estimated heritability for POD activity was 0.51 (Table S2).

\section{QTLs of POD activity identified via GWAS}

The QTLs associated with POD activities were detected through GWAS. A total of 20 QTLs with high reliability $\left(-\log _{10} P \geq 3\right.$ in GWAS and $P<0.05$ in t-test) were detected associated with POD activity (Table 2). The QTLs were distributed on chromosomes 1D, 2A, 2B, 2D,
3A, 3B, 5A, 6B, 7A, and 7B; they explained 5.59\% 12.67\% of the phenotypic variation (PVE) (Table 2).

Five QTL were distributed on Chromosome 2DL, concentrated in the genomic region of $528.60-648.35 \mathrm{Mb}$, which anchors the end of the chromosome close to the telomere. Interestingly, three QTL detected on chromosome $2 \mathrm{AL}$ were also located in the genomic region near the telomere.

We mined the fewest (four) QTL from YuanYang, distributed on chromosomes $2 \mathrm{~A}, 2 \mathrm{~B}, 2 \mathrm{D}$, and $3 \mathrm{~B}$, with a PVE range of $5.62-12.67 \%$. However, $q P O D 2 D .3$ on chromosome 2D $(613.09 \mathrm{Mb})$, contributed the most to PVE (5.62-12.67\%) in this environment. In SQ, we detected 11 QTLs distributed on chromosomes 1D, 2A, $2 \mathrm{~B}, 2 \mathrm{D}, 3 \mathrm{~B}, 5 \mathrm{~A}, 6 \mathrm{~B}$, and $7 \mathrm{~A}$, with a PVE of $6.07-10.16 \%$. 
Table 2 Information for QTL associated with POD activity identified via GWAS

\begin{tabular}{|c|c|c|c|c|c|c|c|}
\hline Num $^{a}$ & QTL & Peak SNP & $\mathrm{Chr}^{\mathrm{b}}$ & Position (bp) & Environment $^{c}$ & $-\log _{10} P$ & $\mathrm{R}^{2}(\%)$ \\
\hline 1 & qPOD1D.1 & AX-109976378 & $1 \mathrm{DL}$ & $462,948,362$ & $\mathrm{SQ}, \mathrm{KF}$ & $3.05 \sim 3.24$ & $7.10 \sim 7.26$ \\
\hline 2 & QPOD2A.1 & AX-108731680 & $2 A S$ & $89,491,698$ & SQ, KF, BLUP & $3.01 \sim 4.47$ & $7.00 \sim 10.16$ \\
\hline 3 & QPOD2A.2 & AX-1111561344 & $2 \mathrm{AL}$ & $711,975,486$ & YY, BLUP & $3.33 \sim 4.19$ & $7.64 \sim 9.77$ \\
\hline 4 & QPOD2A.3 & AX-1111137980 & $2 \mathrm{AL}$ & $752,916,492$ & KF, BLUP & $3.07 \sim 3.68$ & $5.59 \sim 7.19$ \\
\hline 5 & QPOD2A.4 & AX-94872128 & $2 \mathrm{AL}$ & $774,855,749$ & SQ, BLUP & $3.18 \sim 3.33$ & $6.07 \sim 7.38$ \\
\hline 6 & QPOD2B.1 & AX-108880049 & $2 B L$ & $712,656,410$ & YY, BLUP & $3.08 \sim 3.70$ & $7.10 \sim 8.48$ \\
\hline 7 & QPOD2B.2 & AX-110482619 & $2 \mathrm{BL}$ & $790,626,375$ & SQ, BLUP & $3.06 \sim 3.43$ & $6.26 \sim 6.96$ \\
\hline 8 & qPOD2D.1 & AX-111838051 & $2 \mathrm{DL}$ & $528,599,809$ & SQ, BLUP & $3.28 \sim 4.08$ & $7.49 \sim 9.23$ \\
\hline 9 & QPOD2D.2 & AX-108792169 & $2 \mathrm{DL}$ & $571,937,591$ & KF, BLUP & $3.89 \sim 4.22$ & $9.12 \sim 9.73$ \\
\hline 10 & QPOD2D.3 & AX-1111564790 & $2 \mathrm{DL}$ & $613,093,026$ & YY, BLUP & $3.05 \sim 4.59$ & $5.62 \sim 12.67$ \\
\hline 11 & QPOD2D.4 & AX-94769224 & $2 \mathrm{DL}$ & $648,288,487$ & SQ, BLUP & $3.08 \sim 3.17$ & $6.97 \sim 7.30$ \\
\hline 12 & QPOD2D.5 & AX-110105841 & $2 \mathrm{DL}$ & $648,354,189$ & SQ, BLUP & $3.12 \sim 3.63$ & $6.70 \sim 7.96$ \\
\hline 13 & QPOD3A.1 & AX-109586344 & $3 A S$ & $11,834,418$ & KF, BLUP & $3.28 \sim 4.23$ & $7.52 \sim 10.12$ \\
\hline 14 & QPOD3B.1 & AX-95000091 & $3 B S$ & $59,463,829$ & YY, BLUP & $3.12 \sim 3.45$ & $7.11 \sim 7.92$ \\
\hline 15 & qPOD3B.2 & AX-1111774576 & $3 \mathrm{BS}$ & $60,381,223$ & $\mathrm{SQ}, \mathrm{KF}$ & $3.41 \sim 3.50$ & $7.71 \sim 8.23$ \\
\hline 16 & qPOD5A.1 & AX-109025328 & $5 A S$ & $16,976,687$ & $\mathrm{SQ}, \mathrm{KF}$ & $3.58 \sim 3.65$ & $8.10 \sim 8.55$ \\
\hline 17 & qPOD6B.1 & AX-109420494 & $6 \mathrm{BS}$ & $227,564,600$ & $\mathrm{SQ}, \mathrm{KF}$ & $3.09 \sim 3.21$ & $6.90 \sim 7.47$ \\
\hline 18 & QPOD7A.1 & AX-111699555 & $7 \mathrm{AL}$ & $732,587,172$ & KF, BLUP & $3.17 \sim 3.19$ & $7.29 \sim 7.44$ \\
\hline 19 & QPOD7A.2 & AX-109890531 & $7 A L$ & $736,424,469$ & SQ, BLUP & $3.11 \sim 3.51$ & $7.10 \sim 7.92$ \\
\hline 20 & qPOD7B.1 & AX-89436260 & $7 B L$ & $742,059,238$ & KF, BLUP & $3.72 \sim 3.77$ & $7.01 \sim 7.04$ \\
\hline
\end{tabular}

a Num Number

${ }^{\mathrm{b}} \mathrm{Chr}$ Chromosome

'Environment:YY (Yuanyang), SQ (Shangqiu), KF (Kaifeng), BLUP (Best linear unbiased predictor)

In KF, 10 QTLs were distributed on chromosomes 1D, 2A, 2D, 3A, 3B, 5A, 5B, 7A, and 7B (Fig. 2, Table 2), contributing $6.90-10.16 \%$ to phenotypic variation. Among all identified QTL, 16 were found using combined data across the three environments (best linear unbiased predictor, BLUP), while four QTL ( $q P O D 1 D .1$, $q P O D 3 B .2$, $q P O D 5 A .1$, and $q P O D 6 B .1)$ were found only in SQ and KF. One QTL (qPOD2A.1) on chromosome $2 \mathrm{~A}(89.49 \mathrm{Mb})$ was repeatedly detected in three environments, SQ, KF, and BULP (Table 2). Fifteen QTL (four in YY, six in SQ, and five in KF) were screened only in one specific environment and BLUP.

\section{Evaluation of superior and inferior alleles}

For each SNP anchored to the peak of the QTL, the allele contributing to increase POD activity was designated as the superior allele, while the allele that decreased POD activity was inferior. Significant SNP AX-95000091 had the most heterozygous genotypes, at 165 . Five significant SNPs (AX-111137980, AX-94872128, AX-110482619, AX-110105841, and AX-89436260) had two homozygous genotypes and no heterozygous genotypes were detected in the present association population (Table 3).

Six SNPs (AX-108731680, AX-108792169, AX-111564790, AX-94769224, AX-95000091, and
AX-109890531) showed additive effects on POD activity. Three SNPs (AX-108880049, AX-111774576, and AX-111699555) had dominant effect on POD activity $(P<0.05)$. We could not conduct multiple comparisons on six SNPs (AX-109976378, AX-111561344, AX-111838051, AX-109586344, AX-109025328, and AX-109420494), because they had less than two heterozygous genotypes.

Aggregation analysis showed that POD activity was positively correlated with number of superior alleles in the 207 wheat varieties $(r=0.57$, Fig. 3). The more superior alleles a variety contained, the higher its POD activity in the grain. For instance, the variety Shannong26 which containing the highest number of superior alleles (31 alleles) displayed the highest POD activity of $1301.30 \mathrm{U} \cdot \mathrm{min}^{-1} \cdot \mathrm{g}^{-1}$. Similarly, the varieties such as Jimai22 and Yuanzhu which contained 30 superior alleles, showed relatively higher POD activity of $>1000 \mathrm{U} \cdot \mathrm{min}^{-1} \cdot \mathrm{g}^{-1}$ (Table S4).

To benefit genotyping and selection for breeding, we developed two SNPs as KASP markers. One of the KASP was developed from the SNP AX-109420494 which located under the peak of the major QTL - qPOD6B.1 (Table S5). However, for another major QTL, qPOD2A.1, it was failed to develop KASP marker from the SNP 


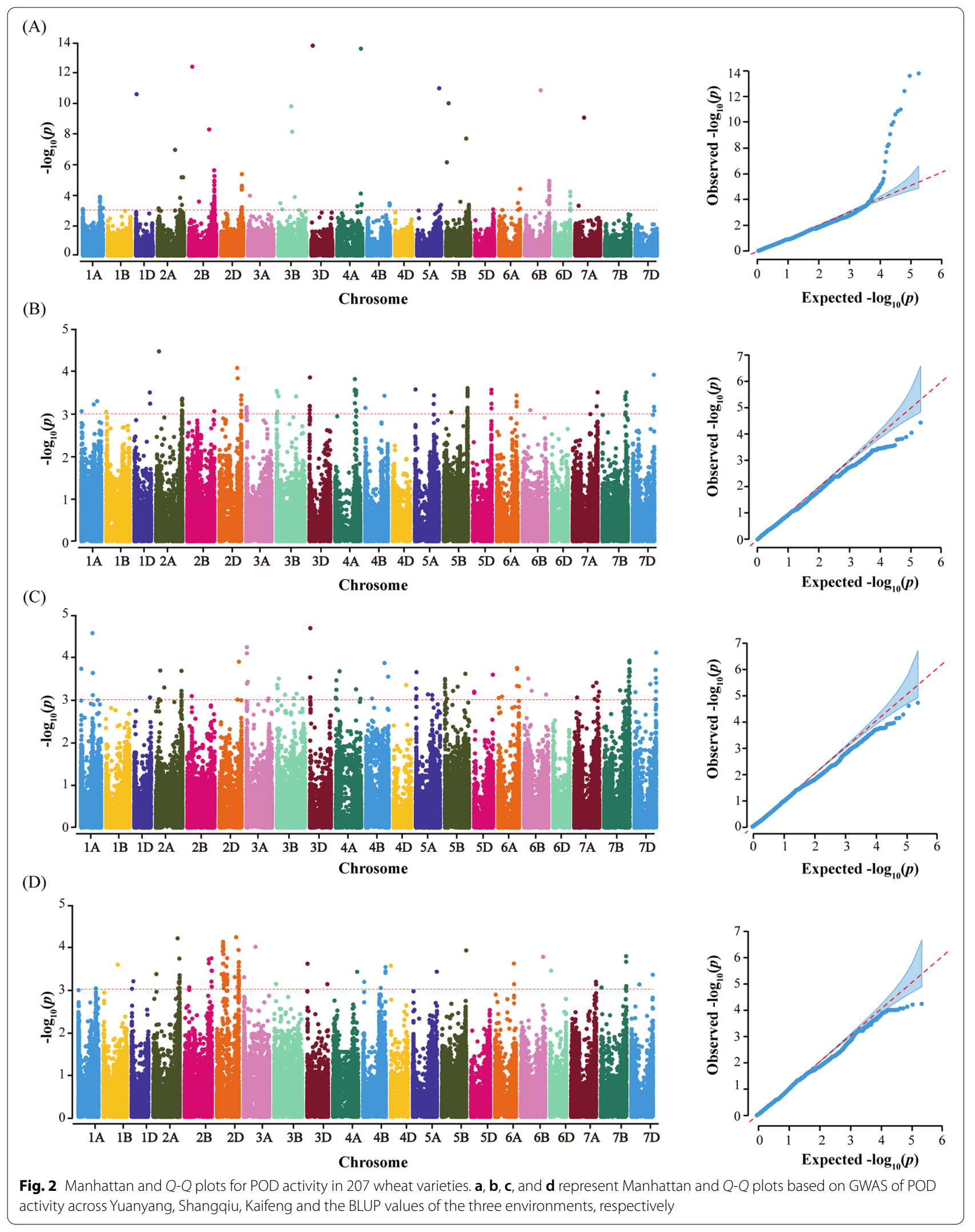


Table 3 Analysis of variance for individuals harboring different genotypes for the 20 SNPs

\begin{tabular}{|c|c|c|c|c|c|c|c|c|c|c|c|}
\hline \multirow{2}{*}{$\frac{\text { Num }}{1}$} & \multirow{2}{*}{$\begin{array}{l}\text { SNP } \\
A X-109976378\end{array}$} & \multirow{2}{*}{$\begin{array}{l}\text { chr } \\
1 D\end{array}$} & \multicolumn{3}{|c|}{ Genotype $^{1}$} & \multicolumn{3}{|c|}{ No. of varieties ${ }^{2}$} & \multicolumn{3}{|c|}{ POD activity $\left(U \cdot \min ^{-1} \cdot \mathrm{g}^{-1}\right)$} \\
\hline & & & CC & $\mathrm{TT}$ & TC & 164 & 42 & 1 & $740.49 b$ & 793.23a & - \\
\hline 2 & AX-108731680 & $2 \mathrm{~A}$ & $\mathrm{CC}$ & $\mathrm{TT}$ & $\mathrm{TC}$ & 178 & 4 & 25 & $738.47 c$ & $1070.34 a$ & $811.95 b$ \\
\hline 3 & AX-111561344 & $2 \mathrm{~A}$ & $\mathrm{TT}$ & CC & $\mathrm{CT}$ & 81 & 121 & 2 & $718.41 b$ & $772.48 a$ & - \\
\hline 4 & $A X-111137980$ & $2 \mathrm{~A}$ & $\mathrm{TT}$ & $\mathrm{CC}$ & None & 64 & 143 & None & $724.45 b$ & $766.87 a$ & None \\
\hline 5 & AX-94872128 & $2 \mathrm{~A}$ & $\mathrm{TT}$ & $\mathrm{CC}$ & - & 18 & 188 & - & $637.27 b$ & $765.95 a$ & - \\
\hline 6 & AX-108880049 & $2 \mathrm{~B}$ & GG & AA & $A G$ & 56 & 4 & 146 & $733.18 b$ & $1122.97 a$ & $751.13 b$ \\
\hline 7 & $A X-110482619$ & $2 \mathrm{~B}$ & $\mathrm{CC}$ & $\mathrm{TT}$ & None & 20 & 187 & None & $637.79 b$ & $766.16 a$ & None \\
\hline 8 & AX-111838051 & $2 \mathrm{D}$ & $\mathrm{CC}$ & GG & GC & 80 & 125 & 2 & $678.68 \mathrm{~b}$ & $798.17 a$ & - \\
\hline 9 & AX-108792169 & $2 \mathrm{D}$ & GG & CC & $C G$ & 158 & 8 & 41 & $760.04 b$ & $1062.85 a$ & $669.23 c$ \\
\hline 10 & AX-1111564790 & $2 \mathrm{D}$ & $\mathrm{CC}$ & $\mathrm{TT}$ & $\mathrm{TC}$ & 137 & 10 & 60 & $759.75 b$ & $942.44 a$ & $708.62 c$ \\
\hline 11 & AX-94769224 & $2 \mathrm{D}$ & $\mathrm{TT}$ & CC & $\mathrm{CT}$ & 19 & 136 & 48 & $633.08 c$ & $781.65 a$ & $722.47 \mathrm{~b}$ \\
\hline 12 & $A X-110105841$ & $2 \mathrm{D}$ & $\mathrm{CC}$ & GG & None & 19 & 188 & None & $633.08 b$ & $765.95 a$ & None \\
\hline 13 & AX-109586344 & $3 \mathrm{~A}$ & $\mathrm{TT}$ & GG & GT & 127 & 78 & 1 & $726.1 \mathrm{~b}$ & $789.04 a$ & - \\
\hline 14 & AX-95000091 & $3 B$ & GG & $\mathrm{CC}$ & $C G$ & 37 & 5 & 165 & $690.65 c$ & 1112.33a & $757.04 b$ \\
\hline 15 & AX-111774576 & $3 B$ & AA & GG & GA & 134 & 3 & 66 & $753.94 b$ & $1104.68 a$ & $731.77 \mathrm{~b}$ \\
\hline 16 & AX-109025328 & $5 \mathrm{~A}$ & GG & $\mathrm{AA}$ & $A G$ & 139 & 66 & 1 & $731.06 \mathrm{~b}$ & $791.12 \mathrm{a}$ & - \\
\hline 17 & AX-109420494 & $6 \mathrm{~B}$ & $\mathrm{TT}$ & GG & GT & 109 & 97 & 1 & $729.74 b$ & $775.41 \mathrm{a}$ & - \\
\hline 18 & AX-111699555 & $7 \mathrm{~A}$ & GG & $\mathrm{TT}$ & TG & 43 & 92 & 70 & $720.42 b$ & $793.51 a$ & $722.94 b$ \\
\hline 19 & AX-109890531 & $7 \mathrm{~A}$ & GG & AA & $A G$ & 89 & 12 & 104 & $698.84 c$ & $744.14 b$ & $803.69 a$ \\
\hline 20 & AX-89436260 & $7 \mathrm{~B}$ & GG & AA & None & 113 & 94 & None & $721.98 b$ & $791.95 a$ & None \\
\hline
\end{tabular}

The values in the column "No. of varieties" and "POD activity" were in turn corresponding to the genotypes in column "Genotype". "None" indicated no such values were detected in association population. "-" represented the uncertainty or missing values. The lowercase letters a, b, and c indicate significant differences after an analysis of variance $(P<0.05)$

1 Genotypes detected from 20 SNPs significantly associated with POD activity. The two letters represented the SNPs from two alleles, respectively

${ }^{2}$ The total variety number may not add up to 207 in cases the genotype of some varieties were missing

AX-108731680 under the peak of the QTL. Therefore, the SNP AX-111134186 which distancing only $0.0056 \mathrm{Mb}$ from the SNP AX-108731680 was selected and successfully developed for KASP marker. Efficiency of the two KASP markers was certified using mean POD activity across the three environments and BLUP. Superior alleles of AX-111134186 and AX-109420494 increased POD activity by $11 \%$ and $6 \%$ compared with inferior alleles, respectively (Table $\mathrm{S6}$ ).

\section{Prediction of candidate genes for POD activity in the association population}

We screened 46 POD-encoding genes within $10 \mathrm{Mb}$ of the sequence flanking QTL ( $5 \mathrm{Mb}$ upstream and downstream), based on genome annotations of 'Chinese Spring' by the International Wheat Genome Sequencing Consortium (IWGSC) and the Wheat Expression Browser (Fig. 4 and Table S7). Interestingly, most genes were highly expressed in roots, while only two genes were highly expressed in grain, these two were TraesCS2B02G615700 (2B:793.46-793.46Mb) and TraesCS2D02G583000 (2D:643.44-643.44Mb), targeting QTL $q P O D 2 B .2$ and $q P O D 2 D .4$, accordingly (Fig. 5a).
The SNPs AX-110482619 (C/T) and AX-94769224 $(\mathrm{T} / \mathrm{C})$ associated with QTLs $q P O D 2 B .2$ and $q P O D 2 D .4$, accordingly, were used for genotyping the 207 tested varieties. Meanwhile, the expression level of TraesCS2B02G615700 and TraesCS2D02G583000, which anchored the corresponding QTL, were detected at 20 days after pollination (DAP) in grains (Table S8). There was no expression divergence of TraesCS2B02G615700 between cultivars with genotype CC and TT in the SNP AX-110482619 locus. Nevertheless, the expression level of TraesCS2D02G583000 between cultivars with genotype TT and CC in the locus AX-94769224 (Fig. 5b, Table S8) were significantly diverged $(P<0.001)$.

TraesCS2D02G583000 expression was used to conduct expression GWAS (eGWAS) and determine whether regulatory element exists in other genetic loci (Fig. 6). Five hundred and five significant SNPs on 20 chromosomes (excluding 4D) in the wheat genome by eGWAS. The PVE ranged from $5.44 \%$ to $15.61 \%$ (Table S9). The significant SNPs screened by eGWAS distributed unevenly on chromosomes. Chromosome 2D concentrated the most number of significant SNPs which the total number was 56 and there were 2 and 54 distributed on the short and long arms, respectively. These SNPs explained 


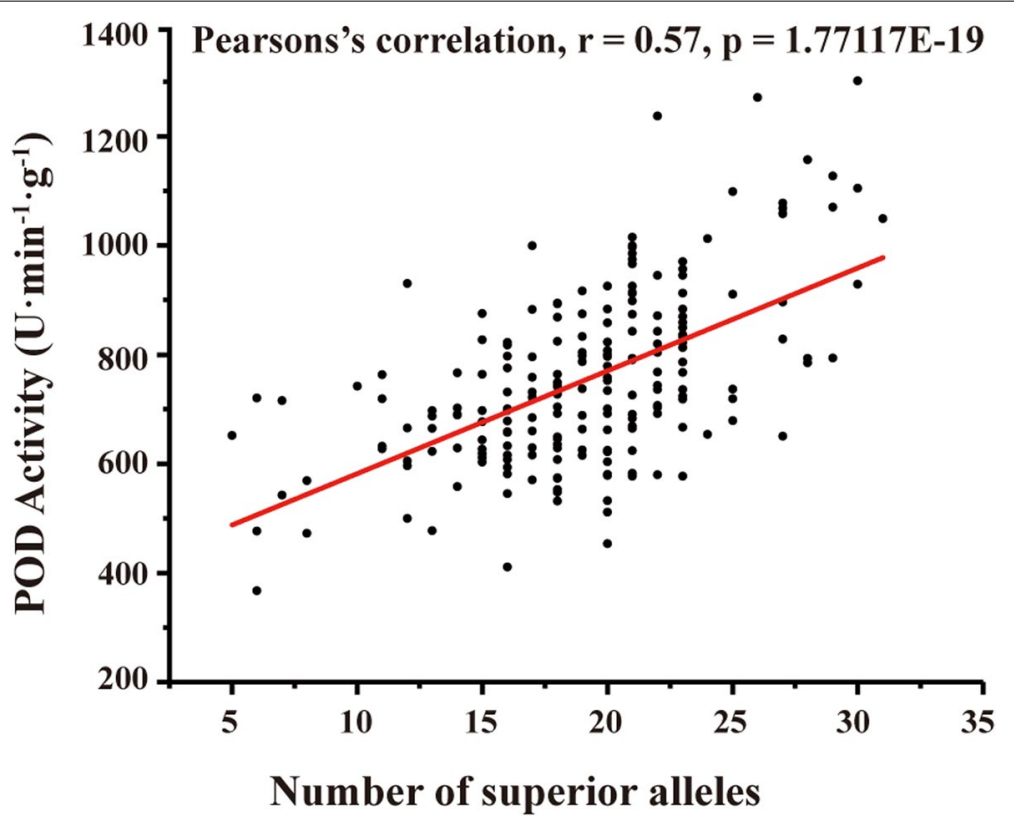

Fig. 3 Correlation analysis between number of superior SNP alleles and POD activity

$6.66-15.55 \%$ of phenotypic variation. Chromosome 3D contained the fewest number (2) of significant eGWAS SNPs. The homelogous group 2 chromosomes (2A, 2B and 2D) were recognized the most important region revealed by eGWAS. The significant SNPs located on homelogous group 2 chromosomes showed relatively higher $-\log _{10} P$ value (3-7.3) than that in other chromosomes and accounted for the highest contribution to the phenotypic variation (5.75-15.61\%) (Table S9). Four SNPs (AX-94872128, AX-110482619, AX-94769224, and AX-110105841) were significantly associated with both POD activity and TraesCS2D02G583000 expression. Nine significant SNPs were within $1 \mathrm{Mb}$ of TraesCS2D02G583000, with AX-108833042 being only $1536 \mathrm{bp}$ away.

\section{Discussion}

In this study, we used a population consisting of 207 wheat varieties to perform a GWAS on POD activity at three locations. We detected 20 QTLs that were significantly associated with POD activity chromosomes $1 \mathrm{D}$, 2A, 2B, 2D, 3A, 3B, 5A, 6B, 7A, and 7B. Previous studies have also identified three of the QTLs ( $q P O D 2 A .4$, $q P O D 2 D .4$, and $q P O D 2 D .5)$, located on chromosomes $2 \mathrm{~A}$ and $2 \mathrm{D}$ [35]. In addition, two QTLs on 2D ( $q$ POD2D.4 and $q P O D 2 D .5)$ were similar to those detected in Gaocheng8901/Zhoumai16 recombinant inbred lines (RILs) by using $90 \mathrm{~K}$ SNP chips [1]. These results indicated a universal function of the locus in 2A and 2D and suggest that the identified SNPs may be part of key loci for POD activity. The panel we used consisted of varieties originating from various planting areas worldwide, with a wide genetic background and variation than populations used in previous studies, which had only Chinese varieties. Thus, we found 17 QTLs associated with POD activity. Our results provide a locus that is more functional for improving POD activity across wide genetic backgrounds and may enhance breeding efficiency.

We then identified 46 POD genes in 20 QTL flanking regions based on the Chinese Spring genome in Ensemble plant database (http://plants.ensembl.org/index. html). Genes related to POD have various biological functions, such as biotic and abiotic stress resistance, lignification, and seed development; they have also been linked to the rheological properties of flour dough. Due to these wide-ranging functions, the expression patterns of different POD genes are divergent in wheat, exhibiting tissue- and developmental stage- specificity. Some genes were highly expressed in all tissues, including coleoptile, root, and embryo in the germinating seed stage; root, crown, and leaf in the seedling stage; and immature inflorescences. Other genes displayed low expression levels. Previous reports have described that POX1, POX2, and POX4 encoded POD were primarily expressed in roots. In our present study, most of the POD genes within $10 \mathrm{Mb}$ of the QTLs were highly expressed in the roots, which was consistent with previous studies [36]. Moreover, two POD genes, TraesCS2B02G615700 and TraesCS2D02G583000, were detected highly expressed in wheat grains. Therefore, we prioritized those SNPs on 


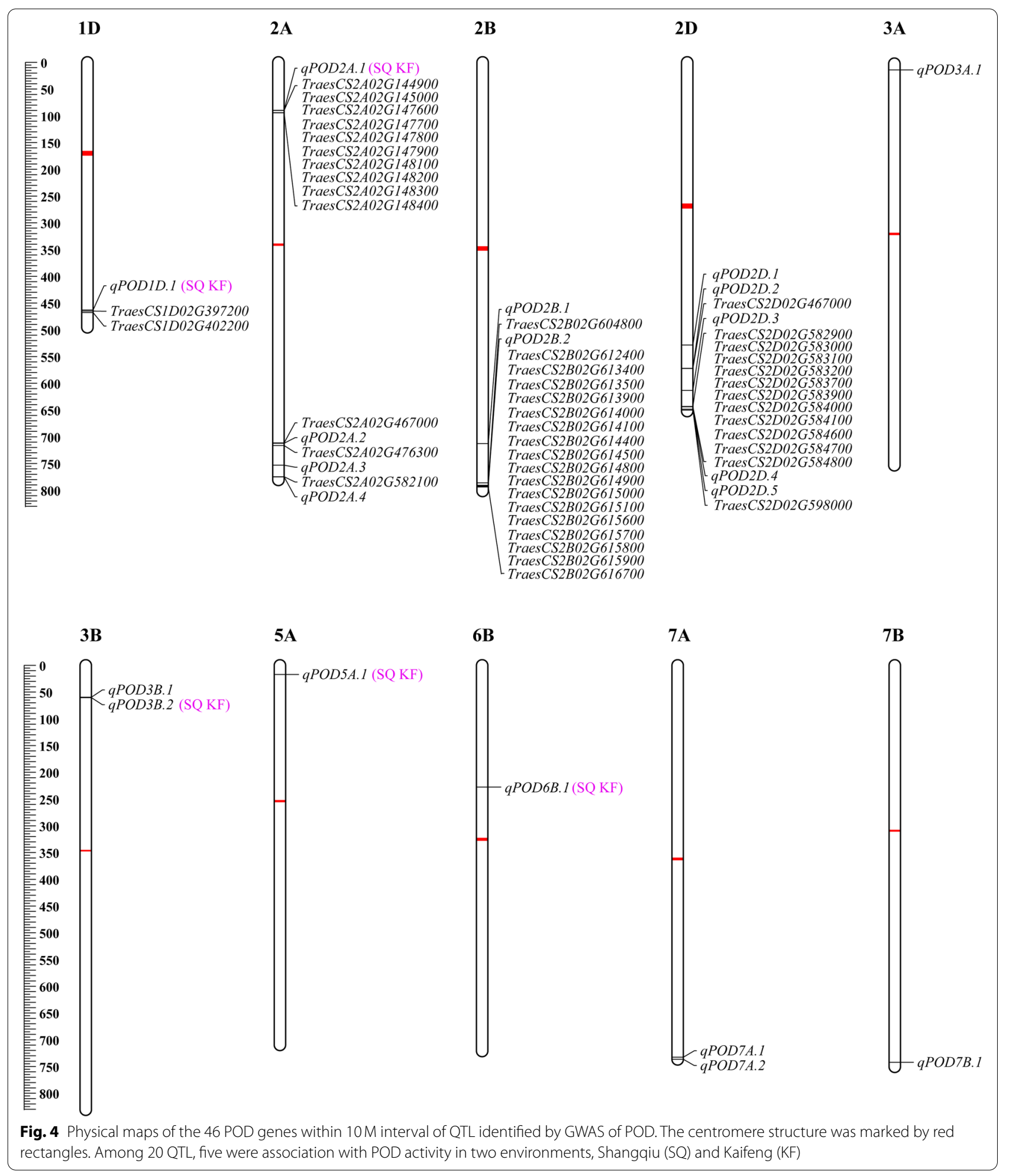

chromosomes 2B and 2D for functional marker development, which should benefit molecular marker-assisted breeding in wheat. Our results revealed that SNPs significantly influence POD activity in the association population, potentially contributing to ubiquitous POD activity in wheat. The eGWAS detected multiple loci, distributed in nearly every chromosome, that influence TraesCS2D02G583000 expression, with of which some 


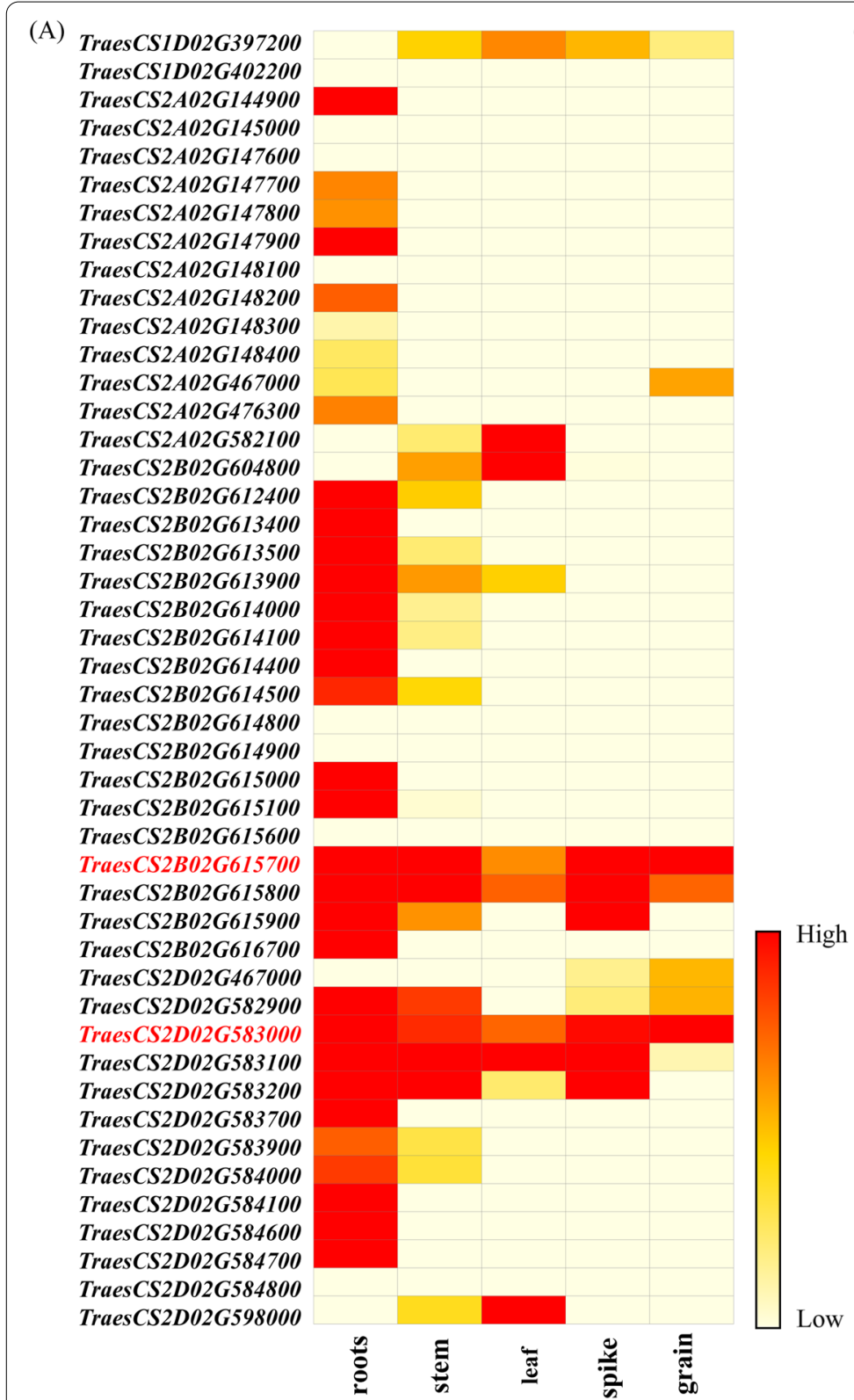

(B)

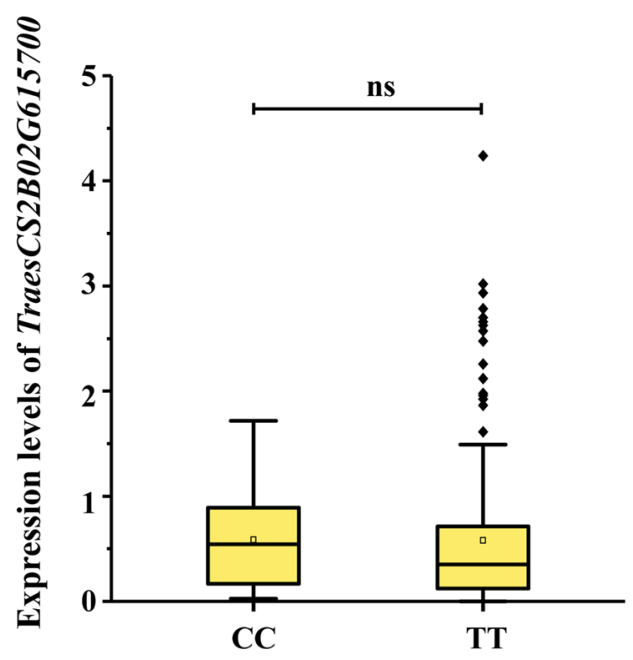

AX-110482619

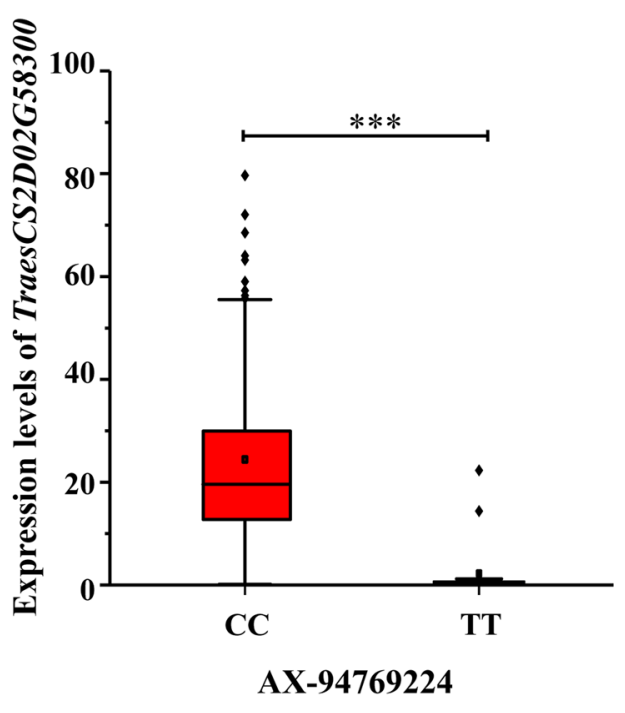

Fig. 5 Prediction of candidate genes for POD activity. a The expression levels of POD genes within $10 \mathrm{M}$ interval of QTL identified by GWAS of POD in different wheat tissues. b The expression levels of TraesCS2B02G615700 and TraesCS2D02G583000 between the cultivars with SNPs AX-110482619 and AX-94769224. ${ }^{* * *}: P<0.001$; ns: no significant

were close to the candidate gene. Therefore, we speculate that both cis- and trans- acting elements may regulate TraesCS2D02G583000 expression. Although our findings provide clues for the molecular mechanisms underlying POD gene regulation, this topic requires further investigation.

Although POD activity is used as a physical and chemical indicator, the detection procedure is cumbersome and time-consuming, given difficulties in measuring all individuals and selecting elite lines from the early generation of breeding populations. Developing and utilizing functional markers is an efficient and economical approach for large-scale screening of high POD activity lines and individuals. Furthermore, based on polymorphisms of functional genes, molecular-assisted breeding has been widely used for wheat [37-39]. However, no molecular markers are available for POD gene selection in breeding, restricting the genetic improvement of 

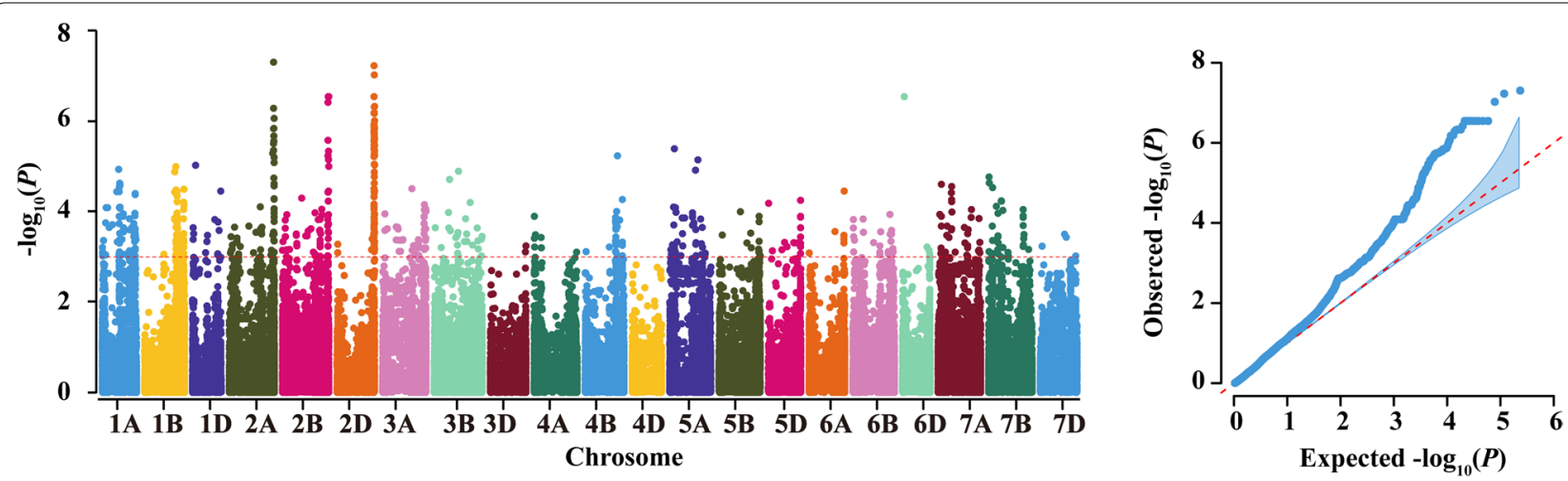

Fig. 6 The Manhattan plots and Q-Q plots of the expression levels of TraesCS2D02G583000

wheat POD activity. Based on two QTL (AX-111134186 and AX-109420494) associated with POD activity on chromosomes $2 \mathrm{~A}$ and $6 \mathrm{~B}$, we developed KASP markers and certified their efficiency in an association population. Further research should yield more KASP markers targeting SNPs associated with POD activity. Combining multiple major genetic sites can significantly improve the efficiency of molecular marker-assisted selection breeding for high POD activity in wheat.

\section{Materials and methods}

\section{Phenotypic variation in the association population}

The population used for GWAS comprised 207 wheat cultivars, which includes typical cultivars from Yellow - Huai Valleys and the Southwestern Wheat Region of China collected by the Quality Breeding Group of Wheat Research Institute, Henan Academy of Agricultural Sciences from Henan Province Crop Germplasm Bank, as well as 7 other countries provided by The International Maize and Wheat Improvement Center (CIMMYT) (Table S1). The authors declare the total permissions to use the collections. Collected cultivars represent a wide range of genetic resources in the world. The population was planted in Yuanyang (E113 $\left.97^{\prime}, \mathrm{N} 35^{\circ} 5^{\prime}\right)$, Shangqiu (SQ, E115 $\left.65^{\prime}, \mathrm{N} 34^{\circ} 45^{\prime}\right)$, and Kaifeng (E114 ${ }^{\circ} 30^{\prime}, \mathrm{N} 34^{\circ} 80^{\prime}$ ) of Henan Province, China, during the 2016-2017 planting season. All cultivars were planted in two rows of $2 \mathrm{~m}$ length, separated by $10 \mathrm{~cm}$ in width and arranged in a randomized complete block. Field management was organized according to local practices at each site.

\section{Phenotypic investigation and data collection}

For each sample, $5 \mu \mathrm{L}$ of POD whole-wheat extract was added to $175 \mu \mathrm{L}$ of a substrate mixture comprising $25 \mu \mathrm{L}$ of $2 \%$ hydrogen peroxide, $5 \mu \mathrm{L}$ of $2 \%$ guaiacol, and $145 \mu \mathrm{L}$ citrate phosphate buffer $(\mathrm{pH}=5.0)$ [32]. Guaiacol in the mixture was catalyzed via the reduction of hydrogen
PODs, taking electrons from hydrogen peroxide and producing a dark-brown product with maximum absorbance at $470 \mathrm{~nm}$. To quantify POD activity, change in absorbance at $470 \mathrm{~nm}$ was measured using a UV-1800PC spectrophotometer (MAPADA). Each sample had two replicates, and their means were used as the phenotype for analysis.

\section{Statistical analysis of phenotypic data}

Descriptive statistics of phenotypic data (POD activity) from each location (Yuanyang, Shangqiu and Kaifeng) was conducted in R version 3.5.1 with the "psych" package. Correlations and variance of phenotypes were analyzed in IBM SPSS Statistics. Broad-sense heritability $\left(H^{2}\right)$ was calculated in R package lme4, according to the formula $H^{2}=\sigma^{2} \mathrm{~g} /\left(\sigma^{2} \mathrm{~g}+\mathrm{o}^{2} \mathrm{ge} / \mathrm{e}+\sigma^{2} \varepsilon / \mathrm{er}\right) \quad\left(\sigma^{2} \mathrm{~g}\right.$ represents genetic variance; $o^{2}$ ge indicates variance of genetic-environmental interaction; $\sigma^{2} \varepsilon$ indicates residual variance; e is number of environments; $r$ is number of replicates in a single environment). Best linear unbiased predictor (BLUP) values were calculated using the formula $\mathrm{Y}=(1 \mid$ Line $)+(1 \mid$ Loc $)+(1 \mid$ Line: Loc) where "Y", " $1 \mid$ ", "Line", and "Loc" represent trait value, groups, all testcrosses, and environments, respectively. These BLUP values for POD activity were used as phenotypic data from an independent environment for further analysis.

\section{Genome-wide association analysis}

Genome-wide association analysis was performed on wheat-grain POD activity in the wheat grain of association population in three environments and BLUP by using the mixed linear model (MLM) [40]. Population structure and kinship based on 224,706 high-quality SNP markers which developed from the Wheat Breeders $660 \mathrm{~K}$ Axiom $^{\circledR}$ array and POD activity of 207 wheat varieties were imported into TASSEL version 5.0 [41, 42]. A threshold of $P$ value at 0.001 was used to determine 
significant marker-trait associations. The false discovery rate (FDR) was calculated using SAS to determine the significance level of the SNP with $P$-value $<0.05$ (Table S10) $[43,44]$. All the SNPs were calculated pair-wise linkage disequilibrium (LD) (Fig. S1) as their squared correlation coefficients $\left(r^{2}\right)$ and LD decay of the whole genome in the association population was evaluated using TASSEL version 5.0. The LD decay was assigned as the corresponding distance at half of maximum $r^{2}$ value. Every stable, significant SNP for POD activity was above the threshold and was associated with more than two environments. Manhattan plots and $Q-Q$ (Quantile-quantile) plots were created using the CMplot code (https://github.com/YinLi Lin/R-CMplot) in R version 3.5.1 [45].

\section{KASP marker development and genotyping}

PolyMarker (http://www.polymarker.info/) was used to design KASP primers. The PCR reaction assay contained primer mix, KASP master Mix and DNA and amplification was performed using the CFX96 Touch $^{\mathrm{TM}}$ real-time PCR detection system. The thermocycling schedule was as follows: initial denaturation at $95^{\circ} \mathrm{C}$ for $15 \mathrm{~min}, 10$ touchdown cycles $\left(95^{\circ} \mathrm{C}\right.$ for $20 \mathrm{~s}$; touchdown at $65^{\circ} \mathrm{C}$ initially and decreasing by $1^{\circ} \mathrm{C}$ per cycle for $\left.25 \mathrm{~s}\right), 30$ additional cycles $\left(95^{\circ} \mathrm{C}\right.$ for $10 \mathrm{~s} ; 57^{\circ} \mathrm{C}$ for $\left.60 \mathrm{~s}\right)$, and three extension steps (each cycle of annealing: $95^{\circ} \mathrm{C}$ for $10 \mathrm{~s}$, $57^{\circ} \mathrm{C}$ for $60 \mathrm{~s}$ ) [46]. At the end of PCR, BIO-RAD automatically determined sample genotype based on position in the allelic discrimination plot. The value of POD activity with different genotypes were calculated according to the method described previously [47].

\section{Candidate gene prediction for POD activity}

Two approaches were used to predict candidate genes for POD activity in associated genomic regions. The first approach referred to gene annotations of the 'Chinese Spring' functional gene database IWGSC (RefSeq version 1.1). All high confidence POD genes within $10 \mathrm{Mb}$ physical intervals from the QTL were used for screening candidate genes. The second approach used gene expression profiling from the Wheat Expression Browser (http:// www.wheat-expression.com); highly expressed genes in the grain were chosen as candidate genes.

\section{Genome-wide association study of POD gene expression}

Grains of 207 cultivars in the association population were used for RNA sequencing at 20 DAP, as previously reported [41]. Peroxidase-gene expression levels (represented as FPKM) were also used as a phenotype for GWAS with a consistent statistics model and threshold. Cis-acting elements were thought to be regulators if a significant SNP site was scanned within $1 \mathrm{Mb}$ interval of the corresponding gene; otherwise, trans-regulation was considered the primary influencer.

\section{Abbreviations}

POD: Peroxidase; MLM: Mixed liner model; GWAS: Genome-wide association study; BLUP: Best linear unbiased predictor; SNP: Single nucleotide polymorphism; Q-Q: Quantile-quantile; PVE: Phenotypic variation explained; IWGSC: International Wheat Genome Sequence Consortium; KASP: Kompetitive AlleleSpecific PCR; QTL: Quantitative trait locus; DAP: Days after pollination.

\section{Supplementary Information}

The online version contains supplementary material available at https://doi. org/10.1186/s12870-021-03299-6.

Additional file 1 : Table S1. The POD activity $\left(U \cdot \mathrm{min}^{-1} \cdot \mathrm{g}^{-1}\right)$ in 207 wheat accessions of each environment and BLUP. Table S2. Analysis of variance (ANOVA) and broad-sense heritability for POD activity based on three environments. Table S3. Marker-trait associations for POD activity in the associated population analyzed by the mixed linear model (MLM). Table S4. Number of superior alleles across 20 significantly associated SNPs in the genome of 207 wheat varieties. Table S5. Primers designed based on KASP markers AX-109420494 and AX-111134186. Table S6. The relationship between superior and inferior alleles of KASP markers to POD activity. Table S7. The expression values of high-confidence POD genes within $10 \mathrm{Mb}$ physical intervals from the significant SNPs. Table S8. The expression of TraesCS2D02G583000 between the varieties in association population based on the genotypes AX-94769224 and AX-110482619. Table S9. The significant SNPs of GWAS analysis conducted by the expression levels of TraesCS2D02G583000. Table S10. The false discovery rate of the 20 significant SNPs.

Additional file $\mathbf{2}$ : Figure $\mathbf{S 1}$. Linkage equilibrium decay plots of $r^{2}$ over physical distance in the association population.

\section{Acknowledgements}

None.

\section{Authors' contributions}

ZF.Z., Z.W. and B. T conceived and designed the experiments; H.G. and ZW.Z. investigated the phenotype; C.L., M.Q. and W.L. conducted the field experiments; Z.L., B. T and Z. W supervised the study; ZF. Z and H. G conducted statistical analysis of the phenotypic data and prepared the figures; S.G., X.S., and C.L. conducted GWAS and prepared the tables; Z.D. J.H. and C.L. analyzed the RNA-seq data and verified gene expression by Real-time PCR; ZF.Z. and H.G. drafted the manuscript, ZF.Z., J.H. and H.G. revised and edited the manuscript. All authors discussed the results and contributed to the manuscript. All authors have read and approved the final manuscript.

\section{Funding}

This research was funded by the National Key Research and Development Program of China (2017YFD0100704) for GWAS population construction and SNPS mining; the Scientific and Technological Research Project of Henan Province (2021021 10027) supported phenotypic investigation; the Agriculture Research System of Henan Province (S2010-01) and the Scientific and Technological Innovation Project of Henan Academy of Agricultural Sciences (2020CX01) for materials planting under different environments across 2 years; Outstanding youth fund of Henan Academy of Agricultural Sciences (2021 JQ04) supported transcript level detection for the candidate genes.

\section{Availability of data and materials}

The datasets necessary for supporting the results of this manuscript are included in this manuscript (and its supplementary files). The RNA-seq data are available in Genome Sequence Archive (https://bigd.big.ac.cn/gsa/ browse/CRA004223). 


\section{Declarations}

Ethics approval and consent to participate

All methods were in compliance with relevant institutional, national, and international guidelines and legislation.

\section{Consent for publication}

Not applicable.

\section{Competing interests}

The authors have declared that no competing interests exist.

\section{Author details}

${ }^{1}$ Henan Institute of Crop Molecular Breeding, Postgraduate T \& R Base of Zhengzhou University, Henan Academy of Agricultural Sciences, Zhengzhou 450002, China. ${ }^{2}$ Agronomy college, Zhengzhou University, Zhengzhou 450001, China. ${ }^{3}$ National Key Laboratory of Wheat and Maize Crop Science, Henan Agricultural University, Zhengzhou 450002, China.

Received: 8 May 2021 Accepted: 27 October 2021

Published online: 10 November 2021

\section{References}

1. Zhai S, He Z, Wen W, Jin H, Liu J, Zhang Y, et al. Genome-wide linkage mapping of flour color-related traits and polyphenol oxidase activity in common wheat. Theor Appl Genet. 2016;129(2):377-94.

2. Zhang W, Dubcovsky J. Association between allelic variation at the phytoene synthase 1 gene and yellow pigment content in the wheat grain. Theor Appl Genet. 2008;116(5):635-45.

3. Singh A, Reimer S, Pozniak CJ, Clarke FR, Clarke JM, Knox RE, et al. Allelic variation at Psy1-A1 and association with yellow pigment in durum wheat grain. Theor Appl Genet. 2009;118(8):1539-48.

4. Chen J, Zhang F, Zhao C, Lv G, Sun C, Pan Y, et al. Genome-wide association study of six quality traits reveals the association of the TaRPP13L1 gene with flour colour in Chinese bread wheat. Plant Biotechnol J. 2019;17(11):2106-22.

5. Baik BK, Czuchajowska Z, Pomeranz Y. Discoloration of dough for oriental noodles. Cereal Chem. 1995;72(2):198-205.

6. Allison SD, Schultz JC. Differential activity of peroxidase isozymes in response to wounding, gypsy moth, and plant hormones in northern red oak (Quercus rubra L.). J Chem Ecol. 2004;30(7):1363-79.

7. Welinder KG. Amino acid sequence studies of horseradish peroxidase. Eur J Biochem. 1979;96(3):483-502.

8. De Gara L. Class III peroxidases and ascorbate metabolism in plants. Phytochem Rev. 2004;3(1):195-205.

9. Dalton DA. Ascorbate peroxidase. Peroxidases Chem Biol. 1991;2:139-53.

10. Welinder K. Bacterial catalase-peroxidases are gene duplicated members of the plant peroxidase superfamily. Biochim Biophys Acta. 1991;1080(3):215-20.

11. Arnison P, Boll W. Isoenzymes in cell cultures of bush bean (Phaseolus vulgaris Cv. Contender): isoenzymatic differences between stock suspension cultures derived from a single seedling. Can J Bot. 1975;53(3):261-71.

12. Jouili H, Bouazizi H, El Ferjani E. Plant peroxidases: biomarkers of metallic stress. Acta Physiol Plant. 2011;33(6):2075.

13. Maksimov IV, Cherepanova EA, Kuzmina OI, Yarullina LG, Akhunov AA. Molecular peculiarities of the chitin-binding peroxidases of plants. Russ J Bioorganic Chem. 2010;36(3):293-300.

14. Zimmerlin A, Wojtaszek P, Bolwell GP. Synthesis of dehydrogenation polymers of ferulic acid with high specificity by a purified cell-wall peroxidase from French bean (Phaseolus vulgaris L.). Biochem J. 1994;299(3):747-53.

15. Fraignier MP, Michaux-Ferriere N, Kobrehel K. Distribution of peroxidases in durum wheat (Triticum durum). Cereal Chem. 2000;77(1):11-7.

16. Fry SC. Cross-linking of matrix polymers in the growing cell walls of angiosperms. Annu Rev Plant Physiol. 1986;37(1):165-86.

17. Taha SA, Sagi F. Relationships between chemical composition of durum wheat semolina and macaroni quality. II. Ash, carotenoid pigments and oxidative enzymes. Cereal Res Commun. 1987;15:123-9.
18. Kobrehel K, Laignelet B, Feillet P. Study of some factors of macaroni brownness. Cereal Chem. 1974;51(5):675-83.

19. Hemalatha MS, Manu BT, Bhagwat SG, Leelavathi K, Prasada Rao UJS. Protein characteristics and peroxidase activities of different Indian wheat varieties and their relationship to chapati-making quality. Eur Food Res Technol. 2007;225(3-4):463-71.

20. Iori R, Cavalieri B, Palmieri S. Cathodic peroxidases of durum wheat flour. Cereal Chem. 1995;72(2):176-81.

21. Gelinas P, Poitras E, McKinnon CM, Morin A. Oxido-reductases and lipases as dough-bleaching agents. Cereal Chem. 1998;75(6):810-4.

22. Hidalgo A, Brandolini A, Pompei C. Carotenoids evolution during pasta, bread and water biscuit preparation from wheat flours. Food Chem 2010;121:746-51.

23. Labuschagne MT, Coetzee MCB, van Deventer CS. General combining ability of six genotypes of spring wheat (Triticum aestivum) for biscuitmaking quality characteristics. Plant Breed. 1996;115(4):279-81.

24. Parker GD, Langridge P. Development of a STS marker linked to a major locus controlling flour colour in wheat (Triticum aestivum L.). Mol Breed. 2000;6(2):169-74

25. Mares D, Aw C. Mapping components of flour and noodle colour in Australian wheat. Aust J Agric Res. 2001;52(12):1297-309.

26. Feillet $P$, Autran J-C, Icard-Vernière C. Pasta brownness: an assessment. J Cereal Sci. 2000;32(3):215-33.

27. Borrelli GM, De Leonardis AM, Platani C, Troccoli A. Distribution along durum wheat kernel of the components involved in semolina colour. J Cereal Sci. 2008;48(2):494-502.

28. Žilić S, Dodig D, Šukalović V, Maksimovic M, Saratlić G, Skrbic B. Bread and durum wheat compared for antioxidants contents, and lipoxygenase and peroxidase activities. Int J Food Sci Technol. 2010;45(7):1360-7.

29. McDonald CE. Lipoxygenase and lutein bleaching activity of durum wheat semolina. Cereal Chem. 1979:56(2):84-9.

30. Brenchley R, Spannagl M, Pfeifer M, Barker GLA, D'Amore R, Allen AM, et al. Analysis of the bread wheat genome using whole-genome shotgun sequencing. Nature. 2012;491(7426):705-10.

31. Kobrehel K, Feillet P. Identification of genomes and chromosomes involved in peroxidase synthesis of wheat seeds. Can J Bot. 2011;53(20):2336-44.

32. Wei J, Geng H, Zhang Y, Liu J, Wen W, Zhang Y, et al. Mapping quantitative trait loci for peroxidase activity and developing gene-specific markers for TaPod-A1 on wheat chromosome 3AL. Theor Appl Genet. 2015;128(10):2067-76.

33. Takasaki S, Kato Y, Murata M, Homma S, Kawakishi S. Effects of peroxidase and hydrogen peroxide on the dityrosine formation and the mixing characteristics of wheat-flour dough. J Agric Chem Soc Japan. 2005;69(9):1686-92.

34. Rebordinos L, Pérez De La Vega M. The inheritance of seed peroxidases of wheat and rye: further data. Theor Appl Genet. 1987;74(6):767-72

35. Jia S, Shengnan Z, Jindong L, Jingxin W, Lu B, Wenwei G, et al. Genomewide association study of grain peroxidase activity in common wheat. Entia Agric Sinica. 2017;50(21):4212-27.

36. Båga M, Chibbar RN, Kartha KK. Molecular cloning and expression analysis of peroxidase genes from wheat. Plant Mol Biol. 1995;29(4):647-62.

37. Bagge $M, X i a X, L u ̈ b b e r s t e d t T$. Functional markers in wheat - commentary. Curr Opin Plant Biol. 2007;10(2):211-6.

38. Liu Y, He Z, Appels $R$, Xia X. Functional markers in wheat: current status and future prospects. Theor Appl Genet. 2012;125(1):1-10.

39. Yan N, Cai S, Guo B, Mou Y, Zhu J, Chen J, et al. A novel mitochondrial tRNA(Val) T1658C mutation identified in a CPEO family. Mol Vis. 2010;16:1736-42.

40. Yu J, Pressoir G, Briggs WH, Vroh Bi I, Yamasaki M, Doebley JF, et al. A unified mixed-model method for association mapping that accounts for multiple levels of relatedness. Nat Genet. 2005;38(2):203-8.

41. Liu C, Zhou Z, Wu Z, Qin M, Shi X, Wang Y, et al. In-depth genetic analysis reveals conditioning of polyphenol oxidase activity in wheat grains by cis regulation of TaPPO2A-1 expression level. Genomics. 2020;112(6):4690-700.

42. Zhou Z, Shi X, Zhao G, Qin M, Ibba MI, Wang Y, et al. Identification of nove genomic regions and superior alleles associated with Zn accumulation in wheat using a genome-wide association analysis method. Int J Mol Sci. 2020;21(6):1928. 
43. Alqudah AM, Sallam A, Stephen Baenziger P, Borner A. GWAS: fastforwarding gene identification and characterization in temperate cereals: lessons from barley - a review. J Adv Res. 2020;22:119-35.

44. Cai D, Xiao Y, Yang W, Ye W, Wang B, Younas M, et al. Association mapping of six yieldrelated traits in rapeseed (Brassica napus L.). Theor Appl Genet. 2014;127(1):85-96.

45. Fu L, Wu J, Yang S, Jin Y, Liu J, Yang M, et al. Genome-wide association analysis of stem water-soluble carbohydrate content in bread wheat. Theor Appl Genet. 2020;133(10):2897-914.

46. Rasheed A, Wen W, Gao F, Zhai S, Jin H, Liu J, et al. Development and validation of KASP assays for genes underpinning key economic traits in bread wheat. Theor Appl Genet. 2016;129(10):1843-60.
47. Zhou Z, Liu C, Qin M, Li W, Wu Z. Promoter DNA hypermethylation of TaGli- $\gamma-2.1$ positively regulates gluten strength in bread wheat. J Adv Res. 2021. https://doi.org/10.1016/j.jare.2021.06.021.

\section{Publisher's Note}

Springer Nature remains neutral with regard to jurisdictional claims in published maps and institutional affiliations.
Ready to submit your research? Choose BMC and benefit from:

- fast, convenient online submission

- thorough peer review by experienced researchers in your field

- rapid publication on acceptance

- support for research data, including large and complex data types

- gold Open Access which fosters wider collaboration and increased citations

- maximum visibility for your research: over $100 \mathrm{M}$ website views per year

At BMC, research is always in progress.

Learn more biomedcentral.com/submissions 